\title{
Estudo sobre as relações entre Autocontrole e Traços de Personalidade
}

\author{
Autocontrole e Traços de Personalidade
}

Fermino Fernandes Sisto

Fabián Javier Marín Rueda

\section{Resumo}

Este estudo investigou as relações entre o autocontrole e traços de personalidade. Foram aplicadas as Escalas Feminina e Masculina de Autocontrole e a Escala de Traços de Personalidade para Crianças em 606 crianças de ambos os sexos, com idade entre 8 e 10 anos de uma escola pública. Verificaram-se correlações negativas e significativas entre os traços de extroversão e sociabilidade e o autocontrole, tanto nas meninas quanto nos meninos. Nos traços de personalidade psicoticismo e neuroticismo evidenciaram-se correlações positivas e significativas com o autocontrole, também em ambos os sexos. Os grupos extremos formados em razão dos traços de personalidade também foram diferenciados significativamente no autocontrole. Dessa forma, os resultados foram interpretados como evidências de validade para as Escalas Feminina e Masculina de Autocontrole.

Palavras-chave: Validade do teste, Avaliação psicológica, Medidas de atitude.

\section{Studying the relationships between Self-control and Personality Traits}

\begin{abstract}
This study investigated the relationships between self-control and personality traits. The Male and Female Scales of Self-Control and the Personality Traits for Children Scale were applied in 606 children of both sexes, aged from 8 to 10 years old of a public school. Negative and significant correlations were verified between the extroversion and sociability traits and self-control, in both girls and boys. Related to psicoticism and neuroticism traits positive and significant correlations were evidenced with the self-control, also in both genders. The extreme groups formed based on the personality traits were significantly differentiated by the self-control. These results were interpreted as validity evidences for the both scales of self-control.
\end{abstract}

Keywords: Test validity, Psychological assessment, Attitude measurement.

\section{Estudio sobre las relaciones entre Autocontrol y Rasgos de Personalidad}

\section{Resumen}

Este estudio investigó las relaciones entre el autocontrol y rasgos de personalidad. Fueron aplicadas las Escalas Feminina e Masculina de Autocontrole y la Escala de Traços de Personalidade para Crianças en 606 niños de ambos sexos, con edad entre 8 y 10 años de una escuela pública. Fueron verificadas correlaciones negativas y significativas entre los rasgos extroversión y sociabilidad y el autocontrol, tanto en las niñas como en los varones. En los rasgos de personalidad psicotismo e neurotismo se verificaron correlaciones positivas y significativas con el autocontrol, también en los dos sexos. Los grupos extremos formados con base en los rasgos de personalidad también fueron diferenciados significativamente en el autocontrol. De esa forma, los resultados fueron interpretados como evidencias de validez para las Escalas Feminina e Masculina de Autocontrole.

Palabras clave: Validez de test, Evaluación psicológica, Medida de actitud. 


\section{Introdução}

O autocontrole pode ser definido como uma forma de controlar o próprio comportamento, geralmente em situações conflituosas, de acordo com padrões definidos pela sociedade (Martinelli \& Sisto, 2006). É uma variável estudada a partir de várias perspectivas, sendo que cada uma delas foca aspectos específicos desse tipo de comportamento. Porém, as diferentes perspectivas coincidem em atribuir ao autocontrole três características básicas, quais sejam, ter uma forma específica de aquisição, um desenvolvimento claro, e ser composto por uma seqüência definida de sub-comportamentos.

Quanto à forma de aquisição, vários são os processos estudados que poderiam levar ao autocontrole. Dentre eles podem ser citadas as experiências passadas de sucesso ou fracasso (Fry, 1975), a internalização das punições (Aronfreed, 1976) e a observação de modelos (Bandura \& Kupers, 1964; Grusec \& Mischel, 1966; Ross, 1971, por exemplo).

Em relação ao desenvolvimento, segundo Shibutani (1961), o autocontrole se manifestaria quando a criança começa a ter a perspectiva do outro, sendo que o comportamento que se mostra controlado seria apenas uma adaptação às exigências das pessoas que convivem com a criança. Para esse mesmo autor, o autocontrole da criança passaria por dois momentos. O primeiro seria quando a criança se motiva a controlar seu comportamento como uma forma de agradar às pessoas significativas em sua vida, seja para obter a sua aprovação, seja para evitar sua desaprovação. A segunda etapa aconteceria quando a criança tenta se auto-agradar e preservar a sua autoimagem e, para que isso aconteça, seria necessário que, na ausência de uma resposta externa, a criança adquira a capacidade de ter prazer ou desprazer consigo mesma.

Já a seqüência definida de sub-comportamentos relacionados ao autocontrole seria, segundo Bandura (1978), a auto-observação, que dependeria dos valores pessoais e do significado funcional da atividade, o julgamento ou auto-avaliação, que dependeria não só de padrões pessoais desenvolvidos por meio do processo de comparação social, mas também da significação pessoal que a atividade possui para o indivíduo, e o auto-reforço, podendo ser entendido como uma auto-recompensa ou uma auto-punição. Em relação a esse processo, Kanfer (1971) sugere que ele se iniciaria quando uma seqüência qualquer de comportamentos fosse interrompida e a criança fosse levada a prestar atenção ao seu próprio comportamento e ao contexto em que ele se insere. $O$ autor ainda distingue dois tipos de autocontrole. $O$ autocontrole que acontece quando existe uma necessidade imediata de tomada de decisão, e aquele que ocorre quando a criança necessita realizar as operações de auto-monitoração, auto-avaliação e auto-reforço durante um período maior de tempo.

Embora o autocontrole tenha sido pesquisado em vários contextos, como por exemplo na área organizacional (Konopkin, Stepanskii \& Kondrat'eva, 1973; Herman, 1971, dentre outros), e na área clínica (Gold \& Cundiff, 1980; Harris \& Johnson, 1980, por exemplo), a maior quantidade de estudos é encontrada na área da educação (Dutrow \& Houston, 1981; Kearney, 1966; Saltz \& Meade, 1973; Wolfe \& Johnson, 1995, entre outros). Nessa área, vários estudos têm apontado o autocontrole como um bom previsor do desempenho acadêmico. Nesse sentido, para Kearney (1966) o autocontrole seria uma variável que se correlaciona positivamente com 0 desempenho acadêmico. Esses achados são confirmados por Dutrow e Houston (1981), que afirmaram que o autocontrole contribui para o aumento da média das notas. Ao lado disso, estudos como o de Whiteside (1966) apontaram que o baixo desempenho acadêmico pode ser parcialmente determinado pela falta de autocontrole. Já Agostin e Bain (1997) colocaram que o autocontrole seria um dos melhores preditores do sucesso escolar na primeira série do ensino fundamental.

Autores também sugeriram que o treinamento do autocontrole poderia conduzir a uma melhoria no rendimento escolar. Sappington, Fritschi, Sandefer e Tauxe (1980) utilizaram o treinamento do autocontrole para aumentar o desempenho acadêmico e mostraram a eficácia do treinamento como forma de melhorar o rendimento acadêmico dos alunos. Pesquisas mostraram um melhor desempenho em escrita (Harris \& Graham, 1985) e em aritmética (Stevenson \& Fantuzzo, 1986) após treinamento em estratégias de autocontrole.

Embora o autocontrole seja uma variável amplamente pesquisada, principalmente no contexto da educação, no Brasil existe uma dificuldade de avaliação, seja porque instrumentos que se propõem a avaliar esse fenômeno encontram-se em artigos ou trabalhos de mestrado e doutorado, seja pelo fato de que os estudos sobre evidências de validade e os dados de precisão nem sempre são conhecidos, além de não apresentarem informações sobre as normas para se comparar e avaliar as pessoas. Dentro dessa perspectiva, Martinelli e Sisto (2006) construíram a Escalas Feminina de Autocontrole (EFAC) e a Escala Masculina de Autocontrole (EMAC).

As escalas avaliam com precisão dois diferentes níveis de autocontrole (em relação às regras e condutas sociais e em relação a sentimentos e emoções) da criança e do jovem, em distintos contextos sociais, com os quais interage freqüentemente. $O$ estudo inicial para construção das escalas contou com 555 crianças de segunda até a oitava série do ensino fundamental de escolas públicas e 
particulares de várias cidades do interior do estado de São Paulo, com idades entre 7 e 18 anos. Como resultado desse estudo, os autores obtiveram um instrumento padronizado para crianças de 8 a 14 anos.

Num primeiro momento, Martinelli e Sisto (2006) consideraram a necessidade de analisar se as respostas aos itens da escala poderiam estar relacionadas ao sexo. Para isso, realizaram uma análise do funcionamento diferencial dos itens. Do total de itens inicialmente estudados (115), 29 deles indicaram diferenciar o sexo, sendo que 13 foram de alta incidência para as meninas e 16 para os meninos.

Em razão disso, os autores decidiram construir duas escalas, uma para as pessoas do sexo masculino e outra para as do sexo feminino. Os itens para cada escala foram de dois tipos: aqueles que não apresentaram diferenças entre os sexos; para a escala do sexo masculino não foram incluídos os itens nos quais as mulheres apresentaram maior intensidade e para a escala do sexo feminino foram retirados aqueles que privilegiavam os homens.

Para interpretar cada fator, Martinelli e Sisto (2006) levaram em consideração a nucleação das frases, com base no conteúdo semântico. Em ambas as escalas os fatores puderam ser interpretados como autocontrole em relação a regras e condutas sociais (fator 1 na escala masculina e fator 2 na feminina) e autocontrole em relação a sentimentos e emoções (fator 2 na escala masculina e fator 1 na feminina).

Quanto às propriedades psicométricas, as evidências de validade da estrutura interna dos itens da escala feminina mostraram que apenas um item apresentou correlação item-total abaixo de 0,30 , sendo que nenhum item com essa característica foi verificado na escala masculina. Quanto à validade de construto em relação ao desenvolvimento, as análises realizadas tanto com a escala feminina quanto com a masculina sugeriram um possível caráter evolutivo do autocontrole.

Em relação à precisão, ela foi calculada pelo alfa de Cronbach e pelo método de teste-reteste. $\mathrm{Na}$ escala feminina o fator 1 obteve um alfa de 0,81 e o fator 2 de 0,73 . Já na escala masculina o alfa do fator 1 foi de $0,84 \mathrm{e}$ do fator 2 de 0,80 . No caso do teste-reteste, em ambas as escalas os autores concluíram que os fatores possuem uma estabilidade temporal bastante boa, com índices de 0,73 e 0,82 para os fatores 1 e 2 respectivamente para o EFAC e 0,86 e 0,82 para os fatores 1 e 2 respectivamente no EMAC. Em relação à escala como um todo, o EFAC apresentou um coeficiente de correlação de Pearson de 0,86 e o EMAC de 0,84 .

Vale ressaltar que embora as escalas apresentem evidências de validade quanto à estrutura interna e bons índices de precisão, nenhum estudo tem se detido a analisar as relações que os diferentes fatores do autocontrole estabelecem com outras variáveis. Assim, é esperado, considerando as características já mencionadas para cada fator do autocontrole, que ele tenha relações com variáveis como emoções ou mesmo com traços de personalidade. Em outros termos, novas pesquisas devem buscar mais evidências de validade para as Escalas Feminina e Masculina de Autocontrole em diferentes âmbitos. É nesse contexto que este estudo se insere, procurando contribuir para a implementação desse instrumento, tendo como foco os traços de personalidade.

Eysenck e Eysenck (1987) consideram a personalidade como uma hierarquia de traços, definindo-os como tendências duradouras, que determinariam formas de se comportar em diferentes situações. Ainda, os autores reconhecem o papel crítico das forças ambientais e da aprendizagem, ressaltando a necessidade de explicar o efeito variável de uma situação para diferentes indivíduos. Assim, as observações da personalidade nessa concepção deveriam ser feitas a partir dos comportamentos das pessoas. Nesse contexto, Eysenck e Eysenck (1987), a partir da perspectiva da teoria dos traços, apontaram que se constata a existência de uma parte variável e outra constante nas condutas dos sujeitos. A parte constante constituiria um traço, sendo que nenhum ato seria gerado a partir de um traço individualmente. Por outro lado, os traços seriam mutáveis, sendo definidos como tendências de comportamentos que covariam. Assim, vale destacar que a perspectiva teórica de personalidade adotada compreende esse fenômeno como um sistema cognitivo, afetivo e comportamental integrados, que interagem com características inatas, adquiridas, orgânicas e sociais, recebendo contribuições de funções psicológicas e sociais (Sisto, Bueno \& Rueda, 2003; Sisto, Oliveira, Oliveira, Bartholomeu, Oliveira \& Costa, 2004).

Dessa forma, observando o aspecto interativo dos quais a configuração dos traços de personalidade dependem, pode-se pensar que algumas características apresentam intersecções com os fatores do autocontrole anteriormente descritos, uma vez que eles também são construídos a partir da interação da pessoa com o meio e seriam acompanhadas por avaliações que os indivíduos fazem de suas próprias experiências e representações. Assim, o estudo da relação entre ambos os construtos deve ser pensada. Nesse sentido, um instrumento que se propõe a avaliar a personalidade é a Escala de Traços de Pesonalidade para Crianças - ETPC (Sisto, 2004), um instrumento capaz de avaliar quatro tipos de traços nas crianças brasileiras.

O instrumento é constituído por quatro escalas que se propõem a avaliar quatro diferentes traços de personalidade, quais sejam, psicoticismo, neuroticismo, extroversão e sociabilidade. Quanto às propriedades psicométricas, apresenta índices satisfatórios de precisão e evidência de validade para os traços avaliados. Para 
calcular a precisão do teste foi utilizado o coeficiente Spearman-Brown, o coeficiente Guttman, o coeficiente alfa como indicador de consistência interna e o método de teste-reteste.

Para as quatro escalas, o coeficiente de SpearmanBrown variou de 0,79 até 0,92, e Guttman de 0,78 a 0,91. O alfa de Cronbach variou de 0,80 a 0,91 . Quando calculados os coeficientes de consistência interna por idade, separadamente, verificou-se que no traço psicoticismo os coeficientes variaram de 0,73 a 0,96; no de extroversão a variação foi de 0,63 a 0,93 ; no de neuroticismo de 0,64 até 0,80 e, por fim; no traço de sociabilidade, o alfa de Cronbach variou de 0,69 até 0,95.

Para verificar a estabilidade temporal, o instrumento foi aplicado com intervalo de três meses entre uma aplicação e outra. Foi calculada a correlação de Pearson entre as pontuações de cada subescala para cada item e independentemente de idade no traço psicoticismo (variando de 0,70 a 0,92); no traço extroversão (de 0,61 a 0,86); no neuroticismo (de 0,43 a 0,84) e; no traço sociabilidade (de 0,72 a 0,90), indicando uma boa estabilidade temporal, se levada em consideração a pouca quantidade de itens para cada fator.

A escala foi submetida a uma validade fatorial, explicando 49,49\% da variância. Quando analisadas as correlações item-total por escala, foram verificadas correlações acima de 0,30 na maioria dos itens.

Quanto à relação do ETPC com variáveis externas, cinco estudos sobre evidências de validade foram registrados na literatura, sendo quatro deles relatados no manual do teste e um em periódico científico. Assim, os traços de personalidade já foram relacionados com a tensão e a ansiedade avaliadas pelo teste de cores de Lüscher (Sisto, Pacheco, Guerrero \& Urquijo, 2001), com as dificuldades de aprendizagem (Bazi, 2003; Pacheco, 2003), com a percepção viso-motora (Sisto \& cols., 2003) e com o autoconceito (Fernandes, Bartholomeu, Rueda, Suehiro \& Sisto, 2005).

Sisto e colaboradores (2001) estudaram a relação entre - ETPC e o teste de cores de Lüscher. Os resultados evidenciaram correlações significativas entre ambos os testes. Os autores concluíram que os conflitos emocionais, avaliados em razão da tensão e da ansiedade, tenderam a aumentar conforme aumentaram os indícios de psicoticismo, e diminuíram conforme aumentaram os indícios de neuroticismo.

Estudando a relação entre os traços de personalidade neuroticismo e extroversão com as dificuldades de aprendizagem em crianças de segundas e terceiras séries, Bazi (2003) verificou diferenças significativas apenas entre os níveis de dificuldade de aprendizagem e o traço extroversão na segunda série. Nessa mesma linha, Pacheco (2003) procurou verificar se os traços de personalidade diferenciavam os alunos com dificuldade acentuada de aprendizagem na escrita daqueles que não apresentavam tais dificuldades. Os traços que apresentaram diferenças significativas foram 0 neuroticismo e a sociabilidade.

Por sua vez, Sisto e colaboradores (2003) investigaram as relações entre os traços de personalidade e a distorção e integração de formas avaliadas pelo Teste de Bender. Os resultados mostraram correlações apenas entre o neuroticismo e algumas medidas de integração, e entre o psicoticismo e as medidas de distorção e uma de integração, sendo elas positivas e significativas. Os autores concluíram que a diminuição da integração visomotora implicaria no aumento do traço neuroticismo, enquanto o aumento da distorção de forma implicaria no aumento do traço psicoticismo.

Posteriormente, o estudo realizado por Fernandes e colaboradores (2005) procurou investigar as relações entre os traços de personalidade e os diferentes tipos de autoconceito, quais sejam escolar, pessoal, social e familiar. Nos meninos foram verificadas correlações positivas e significativas entre a extroversão e os autoconceitos escolar e familiar, entre o neuroticismo e psicoticismo com os autoconceitos pessoal e social, e entre a sociabilidade com o autoconceito pessoal. Também evidenciaram-se correlações negativas e significativas entre a extroversão e o autoconceito social, entre o psicoticismo e o familiar, e entre a sociabilidade e o pessoal. Já no caso das meninas o traço psicoticismo teve correlação positiva com o autoconceito social, e verificaram-se correlações negativas entre extroversão e autoconceito social, psicoticismo e neuroticismo e familiar. Estudando os grupos extremos em relação aos diferentes tipos de autoconceito, os resultados confirmaram os achados anteriormente descritos.

Como pôde ser verificado, tanto os traços de personalidade quanto ao autocontrole são de importância relevante na Psicologia e especialmente no contexto da educação, não sendo verificadas pesquisas na literatura que estabeleçam relações entre ambos os construtos. Dentro desse contexto, este estudo objetivou investigar as relações que podem ser estabelecidas entre traços de personalidade e autocontrole. Esse tipo de informação forneceria evidências de validade para as Escalas Feminina e Masculina de Autocontrole, mais especificamente, evidência de validade de critério concorrente e por grupos extremos. Para realização da pesquisa algumas hipóteses foram aventadas, sendo detalhadas em seguida.

No traço extroversão, característico de pessoas despreocupadas, um tanto agressivas, otimistas, impulsivas e abertas às relações interpessoais, ou seja, sociáveis, há a expectativa que apresente correlações negativas com os tipos de autocontrole, tanto feminino quanto masculino. $O$ mesmo espera-se no traço 
psicoticismo, que caracteriza crianças com uma certa despreocupação em relação aos outros e são solitárias; têm tendência a serem cruéis e insensíveis, mostrando-se hostis, inclusive com os mais íntimos; depreciam o perigo e sentem prazer em perturbar os outros.

Já na sociabilidade são esperadas correlações positivas com o autocontrole, ou seja, a criança com pontuações altas nesse traço tenderiam a apresentar um elevado autocontrole. Por sua vez, no traço neuroticismo são esperadas correlações positivas com os tipos de autocontrole em ambas as escalas. Vale ressaltar que a principal característica de uma criança com alta pontuação em neuroticismo é uma constante preocupação, com forte instabilidade emocional.

Ainda será verificada evidência de validade por grupos extremos em função do ETPC. Dessa forma, as mesmas tendências acima descritas serão.

\section{Método}

\section{Participantes}

Participaram da pesquisa 606 crianças, com idades variando de 8 a 10 anos $(M=9,12, D P=0,782)$, que estavam cursando da $2^{\underline{a}}$ até a $4^{\underline{a}}$ série, de uma escola pública do interior do Estado de São Paulo. Para as crianças que responderam os instrumentos a fim de participarem da pesquisa, não foi adotado nenhum critério de exclusão e/ou inclusão. Do total, 288 (47,5\%) eram meninos e 318 (52,5\%) meninas. De forma geral, a maior freqüência de crianças por idade esteve de acordo com a série que, de fato, freqüentavam. Na segunda e quarta séries houve uma maior concentração de meninas, enquanto que na terceira verifica-se a presença de mais meninos do que meninas. Para uma melhor visualização das idades, série e sexo do total de crianças que participaram da pesquisa, é apresentada a Tabela 1.

Para estudar a discriminação de medidas, as crianças com pontuações extremas (superiores e inferiores) na Escala de Traços de Personalidade para Crianças foram separadas, ficando dessa forma configurado um novo grupo para o estudo. Assim, nos dois grupos de pontuações extremas $(25 \%$ de crianças com menor pontuação e $25 \%$ de crianças com a maior pontuação) em relação a cada um dos traços de personalidade, por meio das medidas fornecidas pela padronização do instrumento, a idade média foi 8,87 com um desvio padrão de 1,15, de um total de 298 crianças.

\section{Instrumentos}

Escalas Feminina e Masculina de Autocontrole (Martinelli \& Sisto, 2006)

Este instrumento é uma escala que se propõe avaliar dois fatores do autocontrole, quais sejam, autocontrole em relação a regras e condutas sociais e autocontrole em relação a sentimentos e emoções, em crianças de 7 a 14 anos. Contém 30 questões na escala feminina, que explicaram $24 \%$ da variância, e 31 questões na masculina explicando $26,77 \%$, que relatam situações que podem ser vivenciadas pelas crianças no seu dia-a-dia, como, por exemplo, "fico muito nervoso quando tenho que falar para a classe" (autocontrole em relação a regras e condutas sociais), "fico irritado quando não sei fazer alguma coisa" (autocontrole em relação sentimentos e emoções).

Foi constituído por meio de uma escala Likert de três pontos (sempre, às vezes e nunca). As questões podem ser positivas ou negativas. Para as positivas, à resposta "sempre" se atribuí 2 pontos, "às vezes", 1 ponto e "nunca", zero pontos. Para as questões negativas, "sempre" corresponde a zero pontos, "às vezes", 1 ponto e "nunca", 2 pontos.

Tabela 1 - Freqüência por idade, sexo e série das crianças participantes da pesquisa.

\begin{tabular}{|c|c|c|c|c|c|}
\hline \multirow{2}{*}{ Série } & \multirow{2}{*}{ Sexo } & \multicolumn{3}{|c|}{ Idade } & \multirow{2}{*}{ Total } \\
\hline & & 8 & 9 & 10 & \\
\hline \multirow{2}{*}{$2^{a}$} & Masculino & 59 & 29 & 2 & 90 \\
\hline & Feminino & 83 & 39 & 4 & 126 \\
\hline \multirow{2}{*}{$3^{a}$} & Masculino & 3 & 88 & 43 & 134 \\
\hline & Feminino & 1 & 79 & 34 & 114 \\
\hline \multirow{2}{*}{$4^{a}$} & Masculino & 0 & 2 & 62 & 64 \\
\hline & Feminino & 0 & 2 & 76 & 78 \\
\hline & Total & 146 & 239 & 221 & 606 \\
\hline
\end{tabular}


Escala de Traços de Personalidade para Crianças ETPC (Sisto, 2004)

É um questionário com 30 itens, que avalia os seguintes traços de personalidade: neuroticismo, psicoticismo, extroversão e sociabilidade. A escala de neuroticismo contém 7 itens; a de extroversão, 10 itens; a de psicoticismo, 11 itens; e a de sociabilidade, 6 itens, sendo que dos 30 itens totais, alguns são encontrados em mais de um traço avaliado. Sua utilização deve ser feita em crianças de 5 a 10 anos de idade. A criança deve responder sim ou não a cada pergunta. A cada sim se atribui um ponto e a cada não zero, sendo o escore do instrumento fornecido pela soma das pontuações nas subescalas. Vale ressaltar que os itens 3 e 26 do psicoticismo são pontuados inversamente, ou seja, à resposta "não" da criança será atribuído 1 ponto e à resposta "sim" 0 ponto. O mesmo procedimento é adotado no item 14 da extroversão e em todos os itens que compõem o traço sociabilidade.

\section{Procedimento}

Após o projeto ser submetido e aprovado pelo Comitê de Ética da Universidade São Francisco, entrou-se em contato com a instituição na qual os dados seriam coletados. Com a autorização da escola procedeu-se à coleta. Os instrumentos foram aplicados coletivamente em sala de aula, por uma equipe com dois estudantes de Psicologia com conhecimento prévio dos testes, um para dar as instruções e o outro para auxiliar as crianças individualmente. Para ambos os testes, cada criança possuía o instrumento com os itens e as alternativas a serem assinaladas. $O$ aplicador lia cada item e esperava que as crianças anotassem sua resposta, fazendo um $x$ na alternativa escolhida, após um breve treinamento para tal.

\section{Resultados}

Todas as análises foram realizadas para cada sexo separadamente. Dessa forma, num primeiro momento são apresentadas as estatísticas descritivas dos traços de personalidade e dos fatores de autocontrole para os sexos masculino e feminino. Posteriormente foram correlacionados os traços de personalidade com os fatores das Escalas Feminina e Masculina de Autocontrole de forma geral e também controlando o efeito da idade e, por fim, os grupos extremos em relação aos traços de personalidade foram comparados por meio da prova $t$ de student. As estatísticas descritivas das medidas trabalhadas na pesquisa encontram-se na Tabela 2.

$\mathrm{Na}$ Tabela 2 observa-se que nos meninos, alguns apresentaram a pontuação máxima possível nos quatro traços de personalidade avaliados pelo ETPC. Nas meninas isso não foi verificado no traço extroversão. Em relação ao autocontrole, os meninos apresentaram maiores pontuações no fator que diz respeito ao autocontrole em relação às regras e condutas sociais, e as meninas tiveram maior freqüência de respostas no fator referente ao autocontrole em relação a sentimentos e emoções.

Para verificar as hipóteses entre os traços avaliados pelo ETPC e o EMAC foram calculados os coeficientes de correlação entre as pontuações em questão, assim como também foi controlado o efeito da idade. Na Tabela 3 podem ser visualizados os resultados obtidos.

Tabela 2 - Estatísticas descritivas do ETPC e das Escalas Feminina e Masculina de Autocontrole.

\begin{tabular}{|c|c|c|c|c|c|c|c|c|}
\hline \multirow{2}{*}{ Medidas } & \multicolumn{4}{|c|}{ Sexo Masculino } & \multicolumn{4}{|c|}{ Sexo Feminino } \\
\hline & $M$ & $D P$ & Mínimo & Máximo & $M$ & $D P$ & Mínimo & Máximo \\
\hline Psicoticismo & 9,92 & 0,99 & 6 & 11 & 10,36 & 0,81 & 6 & 11 \\
\hline Extroversão & 5,79 & 0,84 & 5 & 10 & 5,72 & 0,76 & 5 & 9 \\
\hline Neuroticismo & 5,41 & 0,92 & 0 & 7 & 5,00 & 0,92 & 0 & 7 \\
\hline Sociabilidade & 4,35 & 0,87 & 0 & 6 & 4,00 & 0,86 & 0 & 6 \\
\hline Fator 1 & 22,84 & 4,95 & 6 & 30 & 23,92 & 6,52 & 4 & 40 \\
\hline Fator 2 & 16,28 & 6,00 & 3 & 32 & 15,03 & 3,26 & 2 & 20 \\
\hline Fator Geral & 39,12 & 8,07 & 17 & 60 & 38,95 & 7,81 & 17 & 59 \\
\hline
\end{tabular}


Tabela 3 - Coeficientes de correlação de Pearson e parciais e valores de $p$ entre os traços do ETPC e os fatores do EMAC.

Coeficientes de Correlação de Pearson

\begin{tabular}{|c|c|c|c|c|c|c|c|c|}
\hline & \multicolumn{2}{|c|}{ Psicoticismo } & \multicolumn{2}{|c|}{ Extroversão } & \multicolumn{2}{|c|}{ Neuroticismo } & \multicolumn{2}{|c|}{ Sociabilidade } \\
\hline & $r$ & $p$ & $r$ & $p$ & $r$ & $p$ & $r$ & $p$ \\
\hline Fator 1 & 0,28 & 0,000 & $-0,15$ & 0,010 & 0,14 & 0,017 & $-0,29$ & 0,000 \\
\hline Fator 2 & 0,21 & 0,000 & $-0,11$ & 0,065 & 0,37 & 0,000 & $-0,21$ & 0,000 \\
\hline \multirow[t]{4}{*}{$\begin{array}{l}\text { Escala } \\
\text { Geral }\end{array}$} & 0,33 & 0,000 & $-0,18$ & 0,003 & 0,36 & 0,000 & $-0,33$ & 0,000 \\
\hline & \multicolumn{8}{|c|}{ Coeficientes de Correlação Parcial } \\
\hline & \multicolumn{2}{|c|}{ Psicoticismo } & \multicolumn{2}{|c|}{ Extroversão } & \multicolumn{2}{|c|}{ Neuroticismo } & \multicolumn{2}{|c|}{ Sociabilidade } \\
\hline & $r$ & $p$ & $r$ & $p$ & $r$ & $p$ & $r$ & $p$ \\
\hline Fator 1 & 0,28 & 0,000 & $-0,15$ & 0,010 & 0,14 & 0,016 & $-0,30$ & 0,000 \\
\hline Fator 2 & 0,21 & 0,000 & $-0,11$ & 0,054 & 0,37 & 0,000 & $-0,20$ & 0,001 \\
\hline $\begin{array}{l}\text { Escala } \\
\text { Geral }\end{array}$ & 0,33 & 0,000 & $-0,18$ & 0,003 & 0,36 & 0,000 & $-0,33$ & 0,000 \\
\hline
\end{tabular}

Fator 1 = fator referente ao autocontrole em relação a normas e condutas sociais.

Fator 2 = fator referente ao autocontrole em relação a sentimentos e emoções.

Como mostra a Tabela 3 foram evidenciadas correlações significativas e positivas entre o psicoticismo e o neuroticismo com os fatores do EMAC, bem como negativa com 0 traço sociabilidade. No caso da extroversão não foi verificada correlação significativa apenas no fator que diz respeito aos sentimentos e emoções. Esses dados foram interpretados como o aumento do autocontrole, seja ele em relação às regras ou a sentimentos, corresponde a ele um aumento nos traços de psicoticismo e neuroticismo e diminuição da sociabilidade. $\mathrm{Na}$ extroversão, conforme aumenta a pontuação diminui o autocontrole frente às normas sociais e de forma geral. Em termos da magnitude das correlações verificou-se que as correlações que foram significativas no traço extroversão apresentaram uma magnitude nula, sendo possível observar esse fato também no fator referente a normas e condutas sociais com o traço neuroticismo. O restante das correlações que foram significativas apresentaram uma magnitude considerada baixa. Quando controlado o efeito da idade, a análise indicou que os coeficientes se mantiveram praticamente os mesmos, assim como também as tendências das correlações, ou seja, independentemente da idade há uma associação, mesmo que nula ou baixa, entre os traços avaliados pelo ETPC e os fatores do EMAC.

Também com o intuito de verificar as hipóteses do estudo com os fatores do EFAC foi realizada uma correlação entre as pontuações de ambos instrumentos, controlando também o efeito da idade. Os resultados podem ser observados na Tabela 4.

Da mesma forma que nos meninos, as meninas apresentaram correlações significativas e positivas entre 0 psicoticismo e o neuroticismo com os fatores do EFAC, bem como negativa com 0 traço sociabilidade. $\mathrm{Na}$ extroversão foi constatado que, a diferença dos meninos, o fator referente ao autocontrole em razão das normas e condutas sociais não apresentou níveis de significância.

Os dados, assim como no caso dos meninos, foram interpretados como ao aumento do autocontrole, seja ele em relação às regras ou a sentimentos, Ihe corresponde um aumento nos traços de psicoticismo e neuroticismo e diminuição da sociabilidade. Ao aumento da extroversão Ihe corresponde uma diminuição do autocontrole de forma geral e em relação a sentimentos e emoções. Da mesma maneira que no EFAC, no caso das meninas as magnitudes das correlações apresentaram-se iguais para as correlações que foram significativas.

Por sua vez, a análise da correlação parcial indicou que os coeficientes se mantiveram muito semelhantes ao se controlarem os efeitos da idade, assim como também as tendências das correlações. Dessa forma, também no $E F A C$, independentemente da idade, há uma associação (nula ou baixa) entre os traços avaliados pelo ETPC e os fatores do autocontrole.

Com vistas a verificar até que ponto os grupos extremos em relação aos traços de personalidade se diferenciaram em termos das manifestações de 
autocontrole, esses grupos foram comparados por meio da prova $t$ de student, nível se significância 0,05 , em relação aos fatores fornecidos pelas Escalas Feminina e Masculina de Autocontrole para cada sexo. Os resultados dessa análise para o fator referente às normas e condutas sociais do EMAC e do EFAC encontram-se na Tabela 5.

Tabela 4 - Coeficientes de correlação de Pearson e parciais e valores de p entre os traços do ETPC e os fatores do EFAC.

Coeficientes de Correlação de Pearson

\begin{tabular}{|c|c|c|c|c|c|c|c|c|}
\hline & \multicolumn{2}{|c|}{ Psicoticismo } & \multicolumn{2}{|c|}{ Extroversão } & \multicolumn{2}{|c|}{ Neuroticismo } & \multicolumn{2}{|c|}{ Sociabilidade } \\
\hline & $r$ & $p$ & $r$ & $p$ & $r$ & $p$ & $r$ & $p$ \\
\hline Fator 1 & 0,30 & 0,000 & $-0,14$ & 0,013 & 0,38 & 0,000 & $-0,30$ & 0,000 \\
\hline Fator 2 & 0,25 & 0,000 & $-0,05$ & 0,356 & 0,16 & 0,005 & $-0,27$ & 0,000 \\
\hline \multirow[t]{4}{*}{$\begin{array}{l}\text { Escala } \\
\text { Geral } \\
\end{array}$} & 0,35 & 0,000 & $-0,14$ & 0,014 & 0,38 & 0,000 & $-0,36$ & 0,000 \\
\hline & \multicolumn{8}{|c|}{ Coeficientes de Correlação Parcial } \\
\hline & \multicolumn{2}{|c|}{ Psicoticismo } & \multicolumn{2}{|c|}{ Extroversão } & \multicolumn{2}{|c|}{ Neuroticismo } & \multicolumn{2}{|c|}{ Sociabilidade } \\
\hline & $r$ & $p$ & $r$ & $p$ & $r$ & $p$ & $r$ & $p$ \\
\hline Fator 1 & 0,30 & 0,000 & $-0,14$ & 0,012 & 0,37 & 0,000 & $-0,29$ & 0,000 \\
\hline Fator 2 & 0,24 & 0,000 & $-0,05$ & 0,353 & 0,15 & 0,006 & $-0,26$ & 0,000 \\
\hline $\begin{array}{l}\text { Escala } \\
\text { Geral } \\
\end{array}$ & 0,35 & 0,000 & $-0,14$ & 0,013 & 0,38 & 0,000 & $-0,35$ & 0,000 \\
\hline
\end{tabular}

Fator 1 = fator referente ao autocontrole em relação a sentimentos e emoções.

Fator 2 = fator referente ao autocontrole em relação a normas e condutas sociais.

Tabela 5 - Estatísticas para grupos extremos dos traços de personalidade em relação ao autocontrole referente às normas e condutas sociais do EMAC e do EFAC.

\begin{tabular}{|c|c|c|c|c|c|}
\hline \multirow{2}{*}{$\begin{array}{c}\text { Traços de } \\
\text { Personalidade }\end{array}$} & \multirow{2}{*}{ Grupo } & \multicolumn{4}{|c|}{ Fator 1} \\
\hline & & $M$ & $D P$ & $t$ & $p$ \\
\hline \multicolumn{6}{|c|}{ Sexo Masculino (EMAC) } \\
\hline \multirow[t]{2}{*}{ Psicoticismo } & Baixa pontuação & 21,22 & 5,22 & $-4,46$ & 0,000 \\
\hline & Alta pontuação & 24,07 & 4,62 & & \\
\hline \multirow[t]{2}{*}{ Extroversão } & Baixa pontuação & 24,10 & 4,40 & 3,47 & 0,001 \\
\hline & Alta pontuação & 21,59 & 5,02 & & \\
\hline \multirow[t]{2}{*}{ Neuroticismo } & Baixa pontuação & 21,93 & 5,04 & $-1,99$ & 0,049 \\
\hline & Alta pontuação & 23,35 & 4,63 & & \\
\hline \multirow[t]{2}{*}{ Sociabilidade } & Baixa pontuação & 24,06 & 4,51 & 3,98 & 0,000 \\
\hline & Alta pontuação & 21,19 & 5,23 & & \\
\hline \multicolumn{2}{|c|}{ Sexo Feminino $(E F A C)$} & \multicolumn{2}{|c|}{ Fator 2} & & \\
\hline \multirow[t]{2}{*}{ Psicoticismo } & Baixa pontuação & 14,04 & 3,19 & $-5,33$ & 0,000 \\
\hline & Alta pontuação & 16,15 & 2,74 & & \\
\hline \multirow[t]{2}{*}{ Extroversão } & Baixa pontuação & 15,27 & 2,72 & 1,15 & 0,253 \\
\hline & Alta pontuação & 14,76 & 3,51 & & \\
\hline \multirow[t]{2}{*}{ Neuroticismo } & Baixa pontuação & 14,60 & 3,21 & $-2,04$ & 0,042 \\
\hline & Alta pontuação & 15,45 & 3,47 & & \\
\hline \multirow[t]{2}{*}{ Sociabilidade } & Baixa pontuação & 16,41 & 3,44 & 4,85 & 0,000 \\
\hline & Alta pontuação & 14,22 & 2,84 & & \\
\hline
\end{tabular}


Os grupos extremos da manifestação do autocontrole em relação às normas e condutas sociais no EMAC mostraram diferenças significativas nos quatro traços de personalidade avaliados. Dessa forma, a escala diferenciaria as crianças com mais ou menos características dos traços em questão. Já em relação ao $E F A C$, os traços de personalidade psicoticismo, neuroticismo e sociabilidade apresentaram diferenças significativas, enquanto tais diferenças não foram observadas no traço extroversão. Na Tabela 6 encontramse os resultados referentes ao autocontrole em relação a emoções e sentimentos do EMAC e EFAC.
Como sumariado na Tabela 6 , os traços de personalidade psicoticismo, neuroticismo e sociabilidade apresentaram diferenças significativas nos meninos. Nesse sentido, quem teve pontuação alta no ETPC também teve no fator referente a sentimentos e emoções de autocontrole, e quem teve baixas pontuações em um, também conseguiu no outro. Quanto ao EFAC, verificaramse diferenças significativas nos traços psicoticismo, neuroticismo e sociabilidade, mas não na extroversão. Os resultados dos grupos extremos em relação à escala de autocontrole de forma geral podem ser visualizados na Tabela 7.

Tabela 6 - Estatísticas para grupos extremos dos traços de personalidade em relação ao autocontrole referente a sentimentos e emoções do EMAC e do EFAC.

\begin{tabular}{|c|c|c|c|c|c|}
\hline \multirow{2}{*}{$\begin{array}{c}\text { Traços de } \\
\text { Personalidade }\end{array}$} & \multirow{2}{*}{ Grupo } & \multicolumn{4}{|c|}{ Fator 2} \\
\hline & & $M$ & $D P$ & $t$ & $p$ \\
\hline \multicolumn{6}{|c|}{ Sexo Masculino (EMAC) } \\
\hline \multirow[t]{2}{*}{ Psicoticismo } & Baixa pontuação & 14,59 & 5,31 & $-4,06$ & 0,000 \\
\hline & Alta pontuação & 17,72 & 6,30 & & \\
\hline \multirow[t]{2}{*}{ Extroversão } & Baixa pontuação & 17,36 & 6,23 & 1,54 & 0,125 \\
\hline & Alta pontuação & 15,94 & 6,06 & & \\
\hline \multirow[t]{2}{*}{ Neuroticismo } & Baixa pontuação & 14,33 & 5,45 & $-4,66$ & 0,000 \\
\hline & Alta pontuação & 18,34 & 5,94 & & \\
\hline \multirow[t]{2}{*}{ Sociabilidade } & Baixa pontuação & 18,51 & 6,39 & 3,69 & 0,000 \\
\hline & Alta pontuação & 15,33 & 5,37 & & \\
\hline \multicolumn{2}{|c|}{ Sexo Feminino $(E F A C)$} & \multicolumn{2}{|c|}{ Fator 1} & & \\
\hline \multirow[t]{2}{*}{ Psicoticismo } & Baixa pontuação & 21,25 & 6,52 & $-5,30$ & 0,000 \\
\hline & Alta pontuação & 25,60 & 5,79 & & \\
\hline \multirow[t]{2}{*}{ Extroversão } & Baixa pontuação & 23,78 & 5,36 & 0,62 & 0,535 \\
\hline & Alta pontuação & 23,20 & 7,56 & & \\
\hline \multirow[t]{2}{*}{ Neuroticismo } & Baixa pontuação & 21,78 & 5,13 & $-5,39$ & 0,000 \\
\hline & Alta pontuação & 26,00 & 7,47 & & \\
\hline \multirow[t]{2}{*}{ Sociabilidade } & Baixa pontuação & 26,29 & 7,63 & 4,82 & 0,000 \\
\hline & Alta pontuação & 21,74 & 5,56 & & \\
\hline
\end{tabular}


Tabela 7 - Estatísticas para grupos extremos dos traços de personalidade em relação ao Fator Geral do EMAC e do EFAC.

\begin{tabular}{|c|c|c|c|c|c|}
\hline \multirow{2}{*}{$\begin{array}{c}\text { Traços de } \\
\text { Personalidade }\end{array}$} & \multirow[t]{2}{*}{ Grupo } & \multicolumn{4}{|c|}{ Fator Geral } \\
\hline & & $M$ & $D P$ & $t$ & $p$ \\
\hline \multicolumn{6}{|c|}{ Sexo Masculino (EMAC) } \\
\hline \multirow[t]{2}{*}{ Psicoticismo } & Baixa pontuação & 35,82 & 7,73 & $-5,91$ & 0,000 \\
\hline & Alta pontuação & 41,78 & 7,74 & & \\
\hline \multirow[t]{2}{*}{ Extroversão } & Baixa pontuação & 41,46 & 8,18 & 3,21 & 0,002 \\
\hline & Alta pontuação & 37,53 & 8,08 & & \\
\hline \multirow[t]{2}{*}{ Neuroticismo } & Baixa pontuação & 36,26 & 7,49 & $-4,71$ & 0,000 \\
\hline & Alta pontuação & 41,69 & 7,85 & & \\
\hline \multirow[t]{2}{*}{ Sociabilidade } & Baixa pontuação & 42,56 & 8,05 & 5,20 & 0,000 \\
\hline & Alta pontuação & 36,52 & 7,81 & & \\
\hline \multicolumn{6}{|c|}{ Sexo Feminino $(E F A C)$} \\
\hline \multirow[t]{2}{*}{ Psicoticismo } & Baixa pontuação & 35,29 & 7,39 & $-6,73$ & 0,000 \\
\hline & Alta pontuação & 41,74 & 6,94 & & \\
\hline \multirow[t]{2}{*}{ Extroversão } & Baixa pontuação & 39,04 & 6,62 & 0,99 & 0,325 \\
\hline & Alta pontuação & 37,95 & 8,77 & & \\
\hline \multirow[t]{2}{*}{ Neuroticismo } & Baixa pontuação & 36,38 & 6,46 & $-5,40$ & 0,000 \\
\hline & Alta pontuação & 41,45 & 8,66 & & \\
\hline \multirow[t]{2}{*}{ Sociabilidade } & Baixa pontuação & 42,70 & 7,93 & 6,43 & 0,000 \\
\hline & Alta pontuação & 35,96 & 6,64 & & \\
\hline
\end{tabular}

Quanto ao EMAC de forma geral, verificou-se que todos os traços apresentaram diferenças significativas. Já no $E F A C$, esse resultado não foi verificado apenas para o traço extroversão. Vale ressaltar que pelos dados descritos nas Tabelas 5, 6 e 7, a evidência de validade por grupos extremos foi verificada.

\section{Discussão}

Este estudo foi proposto com base na possibilidade do autocontrole relacionar-se aos traços de personalidade. Assim, algumas hipóteses foram aventadas para pesquisar possíveis associações. Pelas análises realizadas verificouse que a pontuação foi similar entre as meninas e os meninos nas escalas de autocontrole, assim como também nos traços de personalidade avaliados pelo ETPC.

Dentre as hipóteses estudadas, a primeira referia-se ao traço extroversão, no qual esperava-se correlação negativa com o autocontrole, levando em consideração as características de espontaneidade, abertura a relações, otimismo, dentre outras, comuns a essas pessoas. Dessa forma, essas crianças altamente extrovertidas tenderiam a apresentar um baixo autocontrole, seja em relação às regras ou aos sentimentos.

Essa hipótese foi confirmada parcialmente, já que apenas o fator referente às normas e condutas sociais do $E M A C$ e o fator que diz respeito a sentimentos e emoções do EFAC, assim como também a escala de forma geral manteve correlações negativas com o traço em questão, tanto nas meninas quanto nos meninos. Esse fato indicou que, embora de uma forma geral essas crianças possuam um baixo autocontrole, no caso dos meninos, isso é verificado no autocontrole que se refere às regras $e$ condutas sociais, enquanto que nas meninas refere-se ao autocontrole em relação a sentimentos e emoções. Levando em consideração as definições do traço extroversão, o resultado alcançado pareceria estar claro, uma vez que, ao se sentirem mais otimistas, sociáveis e abertas a novas relações, essas crianças muitas vezes poderão não apresentar um adequado controle dos seus comportamentos. Vale ressaltar que esses achados foram também observados quando controlada a variável idade. No caso dos grupos extremos, apenas o fator referente a condutas sociais e a escala geral do EMAC diferenciaram as crianças no traço em questão, pelo que se sugerem 
mais pesquisas com o intuito de serem aprofundados os achados desta.

Esperava-se também que 0 traço neuroticismo apresentasse correlações positivas com o autocontrole. Com base nas análises realizadas, os resultados confirmaram essa hipótese. Assim, características evidentes de ansiedade, depressão, sentimento de culpa, tristeza e nervosismo tenderam a aumentar o autocontrole, seja ele em relação às regras e condutas sociais ou a sentimentos e emoções, tanto nos meninos quanto nas meninas, e independentemente da idade. Pela análise dos grupos extremos tanto no EMAC quanto no EFAC observou-se que o traço neuroticismo foi diferenciado em ambos os fatores das escalas, assim como também nas escalas de forma geral.

No caso do psicoticismo, crianças com esse traço apresentam características de solidão, despreocupação com os demais, hostilidade, tendência à crueldade, prazer em incomodar os outros, dentre outras. Com base nisso, esperavam-se correlações negativas com o autocontrole. Essa hipótese não foi confirmada tanto nas meninas quanto nos meninos independentemente da idade, não só nas escalas de forma geral, mas também em cada um dos fatores que as compõem. Assim, crianças com as características desse traço tendem a apresentar níveis baixos de autocontrole, seja ele em relação às normas sociais ou aos sentimentos e emoções. Esses dados sugerem que essas crianças não estariam preocupadas em mostrar seus verdadeiros sentimentos diante de determinadas situações socialmente punitivas. No caso dos grupos extremos essas tendências também foram mantidas nos meninos e nas meninas em cada um dos fatores assim como também nas escalas de forma geral.

Finalmente, foi aventada a hipótese de que baixas pontuações no traço sociabilidade seriam indicativas de um elevado autocontrole, pois a tendência a não se comportar de acordo com as regras sociais contribuiria para que essas crianças tivessem um autocontrole das suas emoções e comportamentos, não se revelando como elas realmente são. Essa hipótese também foi confirmada tanto nos meninos quanto nas meninas, assim como também independentemente da idade. Assim, quanto menor o respeito às normas sociais dessas crianças, maior a tendência de se auto-controlarem. Os grupos extremos confirmaram esses achados.

Ainda, ressalta-se que com base na magnitude das correlações encontradas concluiu-se que ambos os construtos estudados podem ser considerados discriminantes, uma vez que a comunalidade entre eles variou de $2 \%$ aproximadamente até $14 \%$ aproximadamente. Com base no descrito até aqui, este estudo constatou as evidências de validade a que se propôs. No entanto, sugerem-se novos estudos com 0 objetivo de aprofundar os achados desta pesquisa.
Acrescenta-se que os aspectos emocionais associados aos traços de personalidade e ao autocontrole poderiam favorecer a compreensão de algumas relações aqui encontradas, pois ambas as variáveis envolvem aspectos dessa natureza, de forma que se faz necessário que novos trabalhos aprofundem mais essas questões, talvez, utilizando a Teoria de Resposta ao Item.

\section{Referências}

Agostin, T. M., \& Bain, S. K. (1997). Predicting early school success with developmental and social skills screeners. Psychology in the Schools, 34(3), 219-228.

Aronfreed, J. (1976). Moral development from the stand-point of a general psychological theory. Em T. Lickona (Org.), Moral development and behavior (pp. 54-69). NewYork: Holt, Rinehart and Winston.

Bandura, A., \& Kupers, C. J. (1964) Transmission of patterns of self-reward through modeling. Journal of Abnormal and Social Psychology, 69, 1-9.

Bandura, A. (1978). The self system in reciprocal determinism. American Psychologist, 33, 344-358.

Bazi, G.A. (2003). As dificuldades de aprendizagem na escrita e suas relações com traços de personalidade e emoções. Tese de Doutorado, não publicada, Programa de Pós-graduação em Educação, Universidade Estadual de Campinas, Campinas.

Dutrow, A. M., \& Houston, C. A. (1981). An analysis of the ralationships of academic success and selected achievement/attitude tests. Trabalho apresentado no Annual Meeting of the Southeastern Association for Community College Research, Orlando, FL.

Eysenck, H. J., \& Eysenck, M. W. (1987). Personalidad y diferencias individuales. Madrid: Ediciones Pirámides.

Fernandes, D. C., Bartholomeu, D., Rueda, F. J. M, Suehiro, A. C. B., \& Sisto, F. F. (2005). Auto concepto y rasgos de personalidad: un estudio correlacional. Psicologia Escolar e Educacional, 9(1), 15-25.

Fry, P. S. (1975). Affect and resistance to temptation. Developmental Psychology, 11, 466-472.

Gold, S. R., \& Cundiff, G. (1980). A procedure for increasing selfreported daydreaming. Journal of Clinical Psychology, 36(4), 923927.

Grusec, J., \& Mischel, W. (1966). Model's characteristics as determinants of social learning. Journal of Personality and Social Psychology, 4, 211-215.

Harris, G., \& Johnson, S. B. (1980). Comparison of individualized covert modeling, self-control desensitization, and study skills training for alleviation of test anxiety. Journal of Consulting and Clinical Psychology, 48(2), 186-194. 
Harris, K. R. \& Graham, S. (1985). Improving learning disabled students' composition skills: self-control strategy training. Learning Disability Quarterly, 8(1), 27-36.

Herman, S. M. (1971). Toward a more authentic manager. Training and Development Journal, 25(10), 8-10.

Kanfer, F. M. (1971). The maintenance of behavior by selfgenerated stimuli and reinforcement. Em A. Jacobs \& L. B. Sacha (Orgs.), The psychology of private events. New York: Academic Press.

Kearney, D. L. (1966). Selected non-intellectual factors as predictors of academic success in junior college intellectually capable students. Tese de Doutorado, University of Southern California. Los Angeles, CA.

Konopkin, O. A., Stepanskii, V. I., \& Kondrat'eva, I. I. (1973). Methodological characteristics of the organization of reaction time experiments. Voprosy Psikhologii, 19(4), 159-164.

Martinelli, S. C., \& Sisto, F. F. (2006). Escalas Feminina e Masculina de Autocontrole. São Paulo: Vetor.

Pacheco, L. M. B. (2003). Comportamento de escolares: aspectos acadêmicos e psicossociais na sala de aula. Tese de Doutorado, Faculdade de Educação da Universidade Estadual de Campinas, Campinas, São Paulo.

Ross, S. A. (1971). A test of the generality of the effects of deviant preschool models. Developmental Psychology, 4, 262-267.

Saltz, E., \& Meade, E. (1973). Verbal control of impulsive behavior in the classroom. Detroit: Wayne State University.
Sappington, A. A., Fritschi, O., Sandefer, D., \& Tauxe, M. (1980). Self-directed study skill programs for students on probation. Journal of Counseling Psychology, 27(6), 616-619.

Shibutani, T. (1961). Society and personality. Englewood Cliffs, N. J.: Prentice-Hall.

Sisto, F. F. (2004) Escala de Traços de Personalidade para Criança. São Paulo: Vetor Editora.

Sisto, F. F., Bueno, J. M. H., \& Rueda, F. J. M. (2003). Traços de personalidade na infância e distorção e integração de formas: um estudo de validade. Psicologia em Estudo, 8, 77-84.

Sisto, F. F., Oliveira, S. M. S., Oliveira, K. L., Bartholomeu, D., Oliveira, J. C. S. \& Costa, O. R. S. (2004). Escala de traços de personalidade para crianças e aceitação social entre pares. Interação, 8, 15-24.

Sisto, F. F., Pacheco, L. M. B., Guerrero, P. V. T., \& Urquijo, S. (2001). La tensión y la ansiedad en los rasgos de personalidad: un estudio exploratório. Acta Psiquiátrica y Psicológica de la América Latina, 47(4), 340-350.

Stevenson, H. C., \& Fantuzzo, J. W. (1986). The generality and social validity of a competency-based self-control training intervention for underachieving students. Journal of Applied Behavior Analysis, 19(3), 269-276.

Whiteside, R. (1966). A study of methods of controlling impulses. Abilene, Tex.: Abilene Christian College.

Wolfe, R. N., \& Johnson, S. D. (1995). Personality as a predictor of college performance. Educational and Psychological Measurement, 55(2), 177-185.

Recebido em: 27/11/2007

Revisado em: 09/10/2008

Aprovado em: 21/10/2008

\section{Sobre os autores}

Fermino Fernandes Sisto (fermino.sisto@saofrancisco.edu.br) é Doutor pela Universidad Complutense de Madrid, Livre-docente pela Unicamp e docente do curso de Psicologia e do Programa de Pós-Graduação Stricto Sensu em Psicologia, da Universidade São Francisco, campus Itatiba-SP.

Fabián Javier Marín Rueda (marinfabian@yahoo.com.br) é Psicólogo, Mestre em Avaliação Psicológica, doutorando do Programa de PósGraduação Stricto Sensu em Psicologia da Universidade São Francisco. Bolsista CAPES.

Endereço para correspondência:

Rua José Marciano Filho, 9 - Bairro Corintinha, Itatiba - SP

CEP: $13251-420$

e-mail: marinfabian@yahoo.com.br 